Pacific Journal of Mathematics

ON DEFORMATIONS OF COMPLEX COMPACT MANIFOLDS 


\title{
ON DEFORMATIONS OF COMPLEX COMPACT MANIFOLDS WITH BOUNDARY
}

\author{
Garo K. KiREMIDJIAN
}

Let $M^{\prime}$ be a complex Kählr-Einstein maniford of negative scalar curvature. Let $M$ be a relatively compact submanifold of $M^{\prime}$ such that $\operatorname{dim}_{C} M=\operatorname{dim}_{C} M^{\prime}=\mathrm{n}$ and the boundary $b M$ is a $C^{\infty}$ submanifold of $M^{\prime}$ of real dimension $2 n-1$. It is further assumed that the following condition holds: There exists a constant $c>0$ such that for all $\varphi \in C^{0, q}(\bar{M}, \Theta), q=$ $\left.1,2, \quad\left(\left(2 \square^{\prime}-\tilde{\Delta}\right)\right) \varphi, \varphi\right) \geqq c(\varphi, \varphi)$ where $\Theta$ is the holomorphic tangent bundle of $M^{\prime}, C^{p, q}(\bar{M}, \Theta)$ is the space of all $C^{\infty} \Theta$ valued $(p, q)$ forms extendible to a neighborhood of $\bar{M}, \square^{\prime}$ (resp., $\tilde{\Delta}$ ) is the complex (resp., the real) Laplacian on $C^{p, q}(\bar{M}, \Theta)$ and $($,$) is the L_{2}$-inner product.

The main result of this paper is that there exists a universal family of deformations of $M$ whose parameter space is, in general, a Banach analytic set. In the case when $M$ is a compact Riemann surface with boundary it is shown that real analytic families of complex structures on $M$ can be described in terms of an open set in $\boldsymbol{R}^{m}$ where $m$ is the dimension of the reduced Teichmuller space. The proof of this fact is independent of the theory of quasiconformal mappings and Schwarzian derivatives.

The results of the present work are obtained by extending the methods of M. Kuranishi developed for the case of compact manifolds without boundary ([8], [9]). This approach has already been used successfully in the study of complex structures on noncompact manifolds ([6], [7]). The conditions imposed on $M^{\prime}$ (for a large class of such manifolds we refer to [1]) enable us to use effectively the theory of elliptic boundary value problems (see [5], [10], [11]). Thus we have at our disposal Sobolev $k$-norm estimates without loss of derivatives. This is crucial for the proof of Proposition 3.6 where, by using the implicit function theorem for Banach spaces, we conclude that every almost complex structure $M_{\varphi}$ represented by an element $\phi \in C^{0,1}(\bar{M}, \Theta)$ with sufficiently small $k$-norm is isomorphic to an almost complex structure $M_{\psi}$ with $\bar{\partial}^{*} \psi=0$. Moreover, in the proof of our main theorem (Theorem 3.7), the k-norm estimates allow us to use the inverse mapping theorem for Banach spaces and the result of Proposition 3.6 for the actual construction of the universal family.

1. Preliminaries. Let $M^{\prime}$ be an $n$-dimensional Riemannian manifold and $M \subset M^{\prime}$ a subset with nonempty interior such that the boundary $b M$ is a $C^{\infty}$ submanifold of $M^{\prime}$ and $\bar{M}=M \cup b M$ is compact. 
Then any boundary point of $M$ has a neighborhood $U$ with coordinates $\left(x^{\prime}, \cdots, x^{n-1}, y\right)$ such that $U \cap b M=\{y=0\}, U \cap M=\{y<0\}$ and $U \cap\left(M^{\prime}-\bar{M}\right)=\{y>0\}$.

We denote by $C^{r}(\bar{M})$ the space of $C^{\infty}$ differential form of degree $r$ which are axtendible to an open neighborhood of $M$. For each $p \in M$ the Riemannian metric on $M$ induces an inner product in $C^{r}(\bar{M})$ denoted by $\langle,\rangle_{p}$. Then one has the operator $*: C^{r}(\bar{M}) \rightarrow C^{n-r}(\bar{M})$ given by $\varphi \wedge * \psi=\langle\varphi, \psi\rangle d M, \varphi, \psi \in C^{r}(\bar{M})$, where $d M$ is the volume element of $M$. The inner product over $M$ is defined by $(\varphi, \psi)=$ $\int_{M} \varphi \wedge * \psi$. We denote by $\mathscr{L}^{r}$ the completion of $C^{r}(\bar{M})$ in the $L_{2^{-}}$ norm $\|\varphi\|=(\varphi, \varphi)^{1 / 2}$.

Let $d: C^{r}(\bar{M}) \rightarrow C^{r+1}(\bar{M})$ be the exterior derivative and let $d^{*}=$ $-* d *: C^{r}(\bar{M}) \rightarrow C^{r-1}(\bar{M})$ be its formal adjoint. By Stokes' theorem we have that

$$
(d \varphi, \psi)=\left(\varphi, d^{*} \psi\right)+\int_{b M} \varphi \wedge * \psi, \quad \varphi \in C^{r-1}(\bar{M}), \quad \psi \in C^{r}(\bar{M})
$$

Thus, $(d \varphi, \psi)=\left(\varphi, d^{*} \psi\right)$ for all $\varphi$ if and only if $* \psi=0$ on $b M$. In terms of an admissible boundary coordinate system (i.e., $\left(x_{n}^{\prime}, y\right), x_{n}^{\prime}=$ $\left(x^{1}, x^{2}, \cdots, x^{n-1}\right)$ such that for $y=0$ the Riemannian metric is of the form $\left.d s^{2}=\sum_{\alpha, \beta=1}^{n-1} g_{\alpha \beta}\left(x_{n}^{\prime}, 0\right) d x^{\alpha} d x^{\beta}+\left(d x^{n}\right)^{2}\right)$ this condition means

$$
\psi_{i_{1} \cdots i_{r}}\left(x_{n}^{\prime}, 0\right)=0
$$

if some $i_{\gamma}=n$, where $\psi=\sum_{I} \psi_{I} d x^{I}, I=\left\{i_{1}<\cdots<i_{r}\right\}, d x^{I}=d x^{i_{1}} \wedge \cdots \wedge d x^{i_{r}}$ (see [10]). Let $\mathscr{B}^{r}=\left\{\psi \in C^{r}(\bar{M}) \mid * \psi^{\prime}=0\right.$ on $\left.b M\right\}$.

We now consider a linear mapping $A: C^{r}(\bar{M}) \rightarrow C^{r}(\bar{M})$ which, on a coordinate neighborhood $U$, is given by $A \varphi=\sum_{I, J} A_{J}^{I}(x) \varphi_{I}(x) d x^{J}$. The $A_{J}^{I}$ 's are $C^{\infty}$ functions on $U$ and if $x=\left(x^{1}, \cdots, x^{n}\right)$ are the local coordinates of $p \in b M$, then $A_{J}^{I}(x)$ is a $C^{\infty}$ function in a neighborhood of $p$. We further assume that $A$ is self-adjoint and there exists a constant $c>0$ such that $(A \varphi, \varphi) \geqq c\|\varphi\|^{2}$. Then the bilinear form $Q(\phi, \psi)=(d \varphi, d \psi)+\left(d^{*} \varphi, d^{*} \psi\right)+(A \varphi, \psi)$ gives $\mathscr{B}^{r}$ the structure of a prehilbert space. Let $\hat{\mathscr{B}}$ be the completion of $\mathscr{B}^{r}$ in the norm $\|\psi\|_{Q}=$ $Q(\psi, \psi)^{1 / 2}$. The Riesz representation theorem implies that for each $\alpha \in \mathscr{L}^{r}$ there exists a unique $\varphi \in \hat{\mathscr{B}}^{r}$ such that $Q(\varphi, \psi)=(\alpha, \psi)$ for all $\psi \in \hat{\mathscr{B}}^{r}$. The theory developed in [5], [10], and [11] enables us to conclude that $\varphi \in \mathscr{B}^{r}$ whenever $\alpha \in C^{r}(\bar{M})$ and $\|\varphi\|_{k+2} \leqq c_{k}\|\alpha\|_{k}$ where \|\|$_{k}$ is the Sobolev $k$-norm and $c_{k}$ is a constant depending on $k$ only. In this case $\Delta \varphi+A \varphi=\alpha, \Delta=d d^{*}+d^{*} d$ and ${ }^{*} d \varphi=0$ on $b M$. It is easy to verify the last assertion:

For all $\psi \in C_{0}^{r}(M)=\left\{C^{\infty} r\right.$-forms with compact support in $\left.M\right\}$ we have that 


$$
\left(d d^{*} \varphi, \psi\right)+\left(d^{*} d \varphi, \psi\right)+(A \varphi, \psi)=(\alpha, \psi)
$$

Since $C_{0}^{r}(M)$ is dense in $\mathscr{L}^{r}$, this implies

$$
\Delta \phi+A \varphi=\alpha .
$$

Hence $(\Delta \varphi, \psi)+(A \varphi, \psi)=(\alpha, \psi)$ for all $\psi \in \mathscr{B}$ and by Stokes' theorem

$$
\int_{b . Y} \psi \wedge * d \varphi=0 \text { for all } \psi \in \mathscr{S}^{r}
$$

This gives $* d \varphi=0$ on $b M$.

Next we consider the case when $M$ and $M^{\prime}$ are complex manifold and $E \rightarrow M^{\prime}$ is an $m$-dimensional complex vector bundle. We introduce on the fibers of $E$ a hermitian metric which, on a coordinate neighborhood $U$, is expressed by a positive definite hermitian matrix $h$ whose elements depend differentiably (i.e., $C^{\infty}$ ) on $x \in U$. Let $C^{p, q}(\bar{M}, E)$ be the space of $C^{\infty}$ forms of type $(p, q)$ with values in $E$ which are extendible to an open neighborhood of $M$. On a coordinate neighborhood $U$ an element of $C^{p, q}(\bar{M}, E)$ is given by a column vector $\varphi=$ ${ }^{t}\left(\varphi^{1}, \cdots, \varphi^{m}\right)$ whose components are $C^{\infty}$ forms of type $(p, q)$ on $U$. Then we have the ismorphism $*: C^{p, q}(\bar{M}, E) \rightarrow C^{n-q, n-p}(\bar{M}, E)$ locally defined by $* \varphi={ }^{t}\left(* \varphi^{1}, \cdots, * \varphi^{m}\right)$ and the anti-isomorphism $\sharp: C^{p, q}(\bar{M}, E) \rightarrow$ $C^{q, p}\left(\bar{M}, E^{*}\right), \sharp \varphi=\bar{h} \cdot \bar{\varphi}$, where $E^{*}$ is the dual bundle of $E$. We note that $\#$ commutes with *. If $\rho, \dot{s} \in C^{p, q}(\bar{M}, E),{ }^{t} \varphi \wedge * \#$ is a global scalar $(n, n)$-form and we set $(\varphi, \psi)=\int_{M}^{t} \varphi \wedge * * \psi^{\prime}$. Let $\mathscr{C}^{p, q}(E)$ be the completion of $C^{p, q}(\bar{M}, E)$ with respect to the $L_{2}$-norm $\|\varphi\|=(\rho, \varphi)^{1 / 2}$.

The covariant differentiation associated to the connection $\theta$ of the hermitian metric $h$ is given (locally) by $D \varphi=d \varphi+\theta \wedge \varphi$. The local expression of $\theta$ is $h^{-1} \partial h, d=\partial+\bar{\partial}$. For $i r \in C^{r, s}(\bar{M}, E), \phi \in$ $C^{p, q}(\bar{M}, E), r+s=p+q-1$, we have by Stokes' theorem

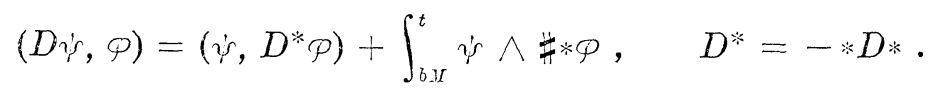

Thus $(D \psi, \varphi)=\left(\psi, D^{*} \phi\right)$ for all $\psi$ if and only if $\varphi \in \mathscr{B}^{p, q}(E)=$ $\left\{\varnothing \in C^{p, q}(\bar{M}, E) \mid * \varphi=0\right.$ on $\left.b M\right\}$. As in the case of scalar differential forms, let $\widetilde{A}: C^{p, q}(\bar{M}, E) \rightarrow C^{p, q}(\bar{M}, E)$ be a continuous linear mapping such that $(\widetilde{A} \varphi, \varphi) \geqq c_{0}\|\varphi\|^{2}$ for all $\varphi$ and some fixed constant $c_{0}>0$, independent of $\varphi$. Then, as before, $\mathscr{S}^{p, q}(E)$ is a prehilbert space with respect to the bilinear form $\widetilde{Q}(\varphi, \psi)=(D \rho, D \psi)+\left(D^{*} \varphi, D^{*} \psi\right)+(\widetilde{A} \psi, \psi)$ and we let $\hat{\mathscr{B}}^{p, q}(E)$ be the completion of $\mathscr{\mathscr { B }}^{p, q}(E)$ with respect to the norm $\|\varphi\|_{\tilde{Q}}=\widetilde{Q}(\varphi, \varphi)^{1 / 2}$. For each $\alpha \in \mathscr{C}^{p, q}(E)$ there exists a unique $\varphi \in \hat{\mathscr{B}}^{p, q}(E)$ such that $\widetilde{Q}(\phi, \psi)=(\alpha, \psi)$ for all $\psi \in \hat{\mathscr{B}}^{p, q}(E)$. Again, the theory developed in [5], [10], and [11] implies that 
$\varphi \in \mathscr{B}^{q, q}(E)$ whenever $\alpha \in C^{p, q}(\bar{M}, E)$ and $\|\varphi\|_{k+2} \leqq \widetilde{c}_{k}\|\alpha\|_{k}$ for some $\widetilde{c}_{k}>0$ depending on $k$ only. Also, in this case we have that $\tilde{\Delta} \phi+$ $\widetilde{A} \varphi=\alpha, \widetilde{\Delta}=D D^{*}+D^{*} D$ and $* D \varphi=0$ on $b M$.

2. Complex structures on complex manifolds. In this section we collect some well known fact about almost complex and complex structures. For a complete discussion we refer to [8] and [9].

We assume now that $M$ is a complex manifold of dimension $n$ and let $M$ be the underlying $C^{\infty}$ manifold of dimension $2 n$. If $C T M$ is the complexification of the real tangent buudle $T M$, then $C T M=$ $\Theta \oplus \bar{\Theta}$ where $\Theta$ is the holomorphic tangent bundle of $M$.

Definition 2.1. An almost complex structure on $X$ is given by a $C^{\infty}$ vector subbundle $\Theta^{\prime}$ of $C T M$ (over $C$ ) such that $C T M=\Theta^{\prime} \oplus \bar{\Theta}^{\prime}$. Let $\rho^{\prime \prime}(M)$ be the projection of $C T M$ onto $\bar{\Theta}$.

Definition 2.2. The almost complex structure $\Theta^{\prime}$ is of the finite distance from the given complex structure $M$ on $M$ if $\rho^{\prime \prime}(M)$ induces an isomorphism from $\bar{\Theta}^{\prime}$ onto $\bar{\theta}$.

The set of almost complex structures of finite distance from $M$ is in bijective correspondence with the set of all $\varphi \in C^{0,1}(M, \Theta)$ such that, at each point $p \in M, \bar{\varphi} \circ \bar{\varphi}: \bar{\Theta}_{p} \rightarrow \bar{\Theta}_{p}$ does not have an eigenvalue 1. An almost complex structure determined by such $\varphi$ will be denoted by $M_{\varphi}$.

Theorem 2.3 (Newlander-Nirenberg). $M_{\varphi}$ is induced by a complex structure if and only if $\bar{\partial} \varphi-[\varphi, \varphi]=0$.

Here $\bar{\partial}$ is the exterior differentiation operator with respect to the complex conjugates of local holomorphic coordinates and $[\varphi, \varphi] \epsilon$ $C^{0,2}(M, \Theta)$ has a local expression

$$
[\varphi, \varphi]=\sum_{a, \beta, \gamma, r}\left(\varphi_{\beta}^{r} \frac{\partial \varphi_{1}^{\alpha}}{\partial z^{r}}-\varphi_{r}^{r} \frac{\partial \varphi_{\beta}^{r}}{\partial z^{r}}\right) d \bar{z}^{\beta} \wedge d \bar{z}^{\gamma} \frac{\partial}{\partial z^{\alpha}}, \quad \varphi=\sum_{\alpha, \beta} \varphi_{\beta}^{\alpha} d \bar{z}^{\beta} \frac{\partial}{\partial z^{\alpha}} .
$$

Let $f$ be a diffeomorphism of $\boldsymbol{M}$ and let $\Theta_{1}^{\prime}$ and $\Theta_{2}^{\prime}$ be almost complex structures on $\boldsymbol{M}$.

DEFINITION 2.4. $f$ is an isomorphism between $\Theta_{1}^{\prime}$ and $\Theta_{2}^{\prime}$ if its differential sends $\Theta_{1}^{\prime}$ to $\Theta_{2}^{\prime}$.

If $\varphi \in C^{0,1}(M, \Theta)$ such that $M_{\varphi}$ can be defined and if $f$ is sufficiently close to the identity map of $M$ in $C^{1}$-topology, then there exists a unique almost complex structure $M_{\omega}$ induced by an element $\omega \in$ $C^{0,1}(M, \Theta)$ such that $f: M_{\omega} \rightarrow M_{\varphi}$ is an isomorphism. We denote $\omega$ by $\varphi \circ f$ and in terms of local coordinates $z=\left(z^{1}, \cdots, z^{n}\right)$ 


$$
\begin{gathered}
\sum_{\beta} \omega_{a}^{\beta}(z)\left(\frac{\partial f^{\gamma}}{\partial z^{\beta}}+\sum_{\mu} \frac{\partial \bar{f}^{\mu}}{\partial z^{\beta}} \phi_{\mu}^{\gamma}(f(z))\right)=\frac{\partial f^{\gamma}}{\partial \bar{z}^{\alpha}}+\sum_{\mu} \frac{\partial \bar{f}^{\mu}}{\partial \bar{z}^{\alpha}} \varphi_{l \prime}^{\gamma}(f(z)), \\
f(z)=\left(f^{1}(z), \cdots, f^{n}(z)\right) .
\end{gathered}
$$

Proposition 2.5. Let $V$ be a normed vector space and let $\xi: V \rightarrow$ $C^{0,0}(M, \Theta)$ be an $\boldsymbol{R}$-linear map. Let $f^{s}(s \in V,|s|$ is small $)$ be a $C^{\infty}$ family of diffeomorphisms of $\boldsymbol{M}$ such that $f^{0}$ is the identity map. Assume that, for each chart $z=\left(z^{1}, \cdots, z^{n}\right)$ of $M$,

$$
f^{\alpha, s}(z) \equiv z^{\alpha}+\xi^{\alpha}(z, s)\left(\bmod |s|^{2}\right)
$$

where $\xi(s)=\sum_{\alpha} \xi^{\alpha}(z, s) \partial /\left(\partial z^{\alpha}\right)$. Let $\varphi \in C^{0,1}(M, \Theta)$ such that $\varphi \circ f^{s}$ can be defined. Then

$$
\varphi \circ f^{s}=\varphi+\bar{\partial}_{\varphi}\left(\xi(s)+\varphi \circ \overline{\xi(s))}+(\bar{\partial} \varphi-[\varphi, \varphi]) \circ \overline{\xi(s)}+0\left(|s|^{2}\right),\right.
$$

where

$$
\begin{aligned}
\bar{\partial}_{\varphi} \xi & =\bar{\partial} \xi-[\varphi, \xi], \quad[\varphi, \xi]=\sum_{\alpha, \beta, \gamma}\left(\frac{\partial \xi^{\beta}}{\partial z^{\gamma}} \varphi_{\alpha}^{\gamma}-\frac{\partial \varphi_{\alpha}^{\beta}}{\partial z^{\gamma}} \xi^{\gamma}\right) d \bar{z}^{\alpha} \frac{\partial}{\partial z^{\beta}}, \\
\varphi \circ \bar{\xi} & =\sum_{\alpha, \beta} \varphi_{\alpha}^{\beta} \bar{\xi}^{\alpha} \frac{\partial}{\partial z^{\beta}} \quad \text { and } \psi \circ \bar{\xi}=\sum_{\alpha, \beta, \gamma} \psi_{\alpha \beta}^{\gamma} \bar{\xi}^{a} d \bar{z}^{\beta} \frac{\partial}{\partial z^{\gamma}}, \\
\psi & =\sum_{\alpha, \beta, \gamma} \psi_{\alpha \beta}^{\gamma} d \bar{z}^{\alpha} \wedge d \bar{z}^{\beta} \frac{\partial}{\partial z^{\gamma}} \in C^{0,2}(M, \Theta) .
\end{aligned}
$$

Proof. Throughout the proof we will make use of the Einstein summation convention. We write $\varphi \circ f^{s}=\varphi+\eta(s)\left(\bmod |s|^{2}\right)$. By $(2.1)$

$$
\begin{aligned}
& \left(\varphi_{\mu}^{\beta}(z)+\eta_{\alpha}^{\beta}(z, s)\right)\left(\delta_{\beta}^{\gamma}+\frac{\partial \xi^{\gamma}(z, s)}{\partial z^{\beta}}+\frac{\overline{\partial \xi^{\mu}(z, s)}}{\partial z^{\beta}} \varphi_{\mu}^{\gamma}(z)\right) \\
\equiv & \frac{\partial \xi^{\gamma}(z, s)}{\partial \bar{z}^{\alpha}}+\left(\delta_{\alpha}^{\prime \prime}+\frac{\partial \bar{\xi}^{\prime \prime}(z, s)}{\partial \bar{z}^{\alpha}}\right) \varphi_{\mu}^{\gamma}(z+\xi(z, s))\left(\bmod |s|^{2}\right), \quad \delta_{\beta}^{\gamma}=\left\{\begin{array}{ll}
1 & \gamma=\beta \\
0 & \gamma \neq \beta
\end{array} .\right.
\end{aligned}
$$

Hence, by collecting terms linear in $s$ we have

$$
\begin{aligned}
& \eta_{\alpha}^{\gamma}(z, s)+\varphi_{\alpha}^{\beta}(z) \frac{\partial \xi^{\gamma}}{\partial z^{\beta}}+\varphi_{\alpha}^{\beta}(z) \frac{\partial \bar{\xi}^{\mu}}{\partial z^{\beta}} \varphi_{\mu}^{\gamma}(z) \\
\equiv & \frac{\partial \xi^{\gamma}}{\partial \bar{z}^{\alpha}}+\frac{\partial \bar{\xi}^{\mu}}{\partial \bar{z}^{\alpha}} \varphi_{\mu}^{\gamma}(z)+\frac{1}{t}\left(\varphi_{\alpha}^{\gamma}(z+\xi(z, t s))-\varphi_{\alpha}^{\gamma}(z)\right)\left(\bmod |s|^{2}\right) \\
\equiv & \frac{\partial \xi^{\gamma}}{\partial \bar{z}^{\alpha}}+\frac{\partial \bar{\xi}^{\mu}}{\partial \bar{z}^{\alpha}} \phi_{\mu}^{\gamma}(z)+\frac{\partial \varphi_{\alpha}^{\gamma}}{\partial z^{\beta}} \xi^{\beta}+\frac{\partial \varphi_{\alpha}^{\gamma}}{\partial \bar{z}^{\beta}} \bar{\xi}^{\beta}\left(\bmod |s|^{2}\right) .
\end{aligned}
$$

Therefore,

$$
\begin{aligned}
& \eta_{\alpha}^{\gamma}(z, s) \\
\equiv & \frac{\partial \xi^{\gamma}}{\partial \bar{z}^{\alpha}}-\varphi_{\alpha}^{\beta}(z) \frac{\partial \xi^{\gamma}}{\partial z^{\beta}}+\frac{\partial \varphi_{\alpha}^{\gamma}}{\partial z^{\beta}} \xi^{\beta}+\varphi_{\mu}^{\gamma}(z) \frac{\partial \bar{\xi}^{\mu}}{\partial \bar{z}^{\alpha}}+\frac{\partial \varphi_{\alpha}^{\gamma} \bar{\xi}^{\beta}-\varphi_{\alpha}^{\beta}(z) \frac{\partial \bar{\xi}^{\mu}}{\partial z^{\beta}} \varphi_{\mu}^{\gamma}(z) .}{\partial \bar{z}^{\beta}} .
\end{aligned}
$$


The sum of the first three terms on the right is the expression of $\bar{\partial}_{\varphi} \xi$. The fourth term is equal to $\partial /\left(\partial \bar{z}^{\alpha}\right)\left(\varphi_{\mu}^{\gamma}(z) \bar{\xi}^{\mu}\right)-\left(\partial \varphi_{\mu}^{\gamma}\right) /\left(\partial z^{\alpha}\right) \bar{\xi}^{\mu}$. The fifth term is equal to

$$
(\bar{\partial} \varphi)_{\beta \alpha}^{\gamma} \bar{\xi}^{\beta}+\frac{\partial \varphi_{\beta}^{\gamma}}{\partial \bar{z}^{\alpha}} \bar{\xi}^{\beta}=[\varphi, \varphi]_{\beta \alpha}^{r} \bar{\xi}^{\beta}+\frac{\partial \varphi_{\beta}^{\gamma}}{\partial \bar{z}^{\alpha}} \bar{\xi}^{\beta}+(\bar{\partial} \varphi-[\varphi, \varphi])_{\beta a}^{\gamma} \bar{\xi}^{\beta} .
$$

Therefore, $\eta=\bar{\partial}_{\varphi} \xi+\bar{\partial}(\varphi \circ \bar{\xi})+\chi+(\bar{\partial} \varphi-[\varphi, \varphi]) \circ \bar{\xi}$, where

$$
\begin{aligned}
\chi_{\alpha}^{\gamma} & =[\varphi, \varphi]_{\beta \alpha}^{\gamma} \bar{\xi}^{\beta}-\varphi_{\alpha}^{\beta}(z) \frac{\partial \bar{\xi}^{\mu}}{\partial z^{\beta}} \varphi_{\mu}^{\gamma}(z) \\
& =\left(\varphi_{\beta}^{\mu}(z) \frac{\partial \varphi_{\alpha}^{\gamma}}{\partial z^{\mu}}-\varphi_{\alpha}^{\mu}(z) \frac{\partial \varphi_{\beta}^{\gamma}}{\partial z^{\mu}}\right) \bar{\xi}^{\beta}-\varphi_{\alpha}^{\mu}(z) \frac{\partial \bar{\xi}^{\beta}}{\partial z^{\mu}} \varphi_{\beta}^{\gamma}(z) \\
& =\varphi_{\beta}^{\mu}(z) \bar{\xi}^{\beta} \frac{\partial \varphi_{\alpha}^{\gamma}}{\partial z^{\mu}}-\varphi_{\alpha}^{\mu}(z) \frac{\partial\left(\varphi_{\beta}^{\gamma} \bar{\xi}^{\beta}\right.}{\partial z^{\mu}}=-[\varphi, \varphi \circ \bar{\xi}]_{\alpha}^{\gamma} .
\end{aligned}
$$

Hence $\eta=\bar{\partial}_{\varphi}(\xi+\varphi \circ \bar{\xi})+(\bar{\partial} \varphi-[\varphi, \varphi]) \circ \bar{\xi}$.

3. Families of complex structures on certin manifolds with boundary. Let $M$ and $M^{\prime}$ be complex manifolds of dimension $n$ such that $M \subset M^{\prime}$, the boundary $b M$ is a $C^{\infty}$ submanifold of $M^{\prime}$ of (real) dimension $2 n-1$ and $\bar{M}=M \cup b M$ is compact. We fix once and for all a sufficiently large integer $k$ with the following property: There exists a constant $c_{k}>0$ such that if $U$ is a coordinate neighborhood of $M$ and if $u_{1}, u_{2}$ are $C^{\infty}$ functions with supp $u_{i}=\left\{x \in M^{\prime} \mid u_{i}(x) \neq 0\right\} \subset$ $U, i=1,2$, then

$$
\left|D^{\ell} u_{i}\right| \leqq c_{k}\left\|u_{i}\right\|_{k}, \quad\left\|u_{1} \cdot u_{2}\right\|_{k} \leqq c_{k}\left\|u_{1}\right\|_{k}\left\|u_{2}\right\|_{k},
$$

for $|\ell|=\ell_{1}+\cdots+\ell_{2 n} \leqq k-n-1$, where $D^{\ell}=\partial^{\ell_{1}+\cdots+\ell_{2 n}} /\left(\partial x^{\ell_{1}} \cdots \partial x_{2 n}^{\ell_{2 n}}\right)$ and \|\|$_{k}$ is the Sobolev $k$-norm over $M$. The existence of such an integer $k$ is guaranteed by Sobolev's lemma.

If $E$ is a vector bundle over $M^{\prime}$ we will denote by $\mathscr{H}_{k}^{p, q}(E)$ the completion of $C^{p, q}(\bar{M}, E)$ in the norm \|\|$_{k}$. It is clear that $\varphi \in C^{0,1}(\bar{M}, \Theta)$ with sufficiently small $\|\varphi\|_{k}$ represents an almost complex structure $M_{\varphi}$ on $\boldsymbol{M}$.

Before stating and proving our main result we will give some definitions.

Definition 3.1. A subset $S$ of a complex Banach space $X$ is a Banach analytic set (or simply an analytic set) if for each $s \in S$ there exists an open neighborhood $\Omega$ of $s$ in $X$, a Banach space $Y$ and an analytic map $g: \Omega \rightarrow Y$ such that $S \cap \Omega=g^{-1}(0)$.

Definition 3.2. If $S$ and $T$ are analytic sets in the Banach spaces $X$ and $Y$, resp., a map $\tau: S \rightarrow T$ is analytic if, for each point 
$s \in S, \tau$ can be extended to an analytic map of an open neighborhood of $s$ in $X$ into $Y$.

DEFINITION 3.3. A complex analytic family of deformations of $M$ is a triple $\left(S, \psi, s_{0}\right)$ where $S$ is an analytic set, $\psi: S \rightarrow C^{0,1}(\bar{M}, \Theta)$ is an analytic map (i.e., the composition of $\psi$ with the inclusion $C^{0,1}(\bar{M}, \Theta) \subset \mathscr{K}_{k}^{0,1}(\Theta)$ is analytic) such that for each $s \in S$, $\psi(s)$ represents a complex structure $M_{\psi(s)}$ on $M$ and $s_{0} \in S$ with $\psi\left(s_{0}\right)=0$.

DEFINITION 3.4. A complex analy tic family $\left(T, \varphi, t_{0}\right)$ is a universal family of deformations of $M$ if for any other family $\left(S, \psi, s_{0}\right)$ there exists a neighborhood $S^{\prime}$ of $s_{0}$ in $S$, an analytic map $\tau: S^{\prime} \rightarrow T$ and a $C^{\infty}$ family of diffeomorphisms $f^{s}$ of $\boldsymbol{M}$ such that $\tau\left(s_{0}\right)=t_{0}, f^{s_{0}}=i d$ and $\psi(s) \circ f^{s}=\varphi(\tau(s))$.

REMARK 3.5. Let $\sigma$ be a Riemannian metric on $M^{\prime}$ with respect to which $b M$ is a totally geodesic submanifold. It is well known that such a metric always exists. Let $\xi \in C^{0,0}(\bar{M}, \Theta)$ such that the real vector field $\xi+\bar{\xi}$ is tangent to $b M$. Then, if $\xi$ is sufficiently small in $C^{1}$-topology, the map $f: M \rightarrow M$ defined by $p \mapsto f(p)$, where $f(p)$ is the end point of the geodesic (with respect to $\sigma$ ) of length $\|\xi+\bar{\xi}\|$ drawn from $p$ to the direction $(\xi+\bar{\xi}) /\|\xi+\bar{\xi}\|$, is a diffeomorphism. It is easy to see that in terms of local coordinates

$$
f^{\alpha}(z)=z^{\alpha}+\xi^{\alpha}(z)\left(\bmod \|\xi\|^{2}\right) .
$$

We will denote the diffeomorphism $f$ by $e(\xi)$.

Proposition 3.6. Assume that $M^{\prime}$ has a Kähler-Einstein metric $g$ of negative scalar curvature. Let $P=\left\{\xi \in C^{0,0}(\bar{M}, \Theta) \mid *(\# \xi+\overline{\# \xi})=\right.$ $* d(\# \xi+\$ \xi)=0$ on $b M\}$ where the operators $\#$ and $*$ are defined with respect to the metric $g$, viewed as a metric on the fibers of $\theta$, and \#s is considered as a scalar form of type $(1,0)$. Then there are neighborhoods of the origin $V$ and $W$ of $C^{0,1}(\bar{M}, \Theta)$ and $P$, respectively, in the \|\|$_{k}$-norm topology so that for each $\phi \in V$ there exists a unique $\xi=\xi(\varphi)$ in $W$ such that $\bar{\partial}^{*}(\varphi \circ e(\xi))=0$, where $\bar{\partial}^{*}=-* \# \bar{o} \neq *$ is the formal adjoint of the operator $\bar{\partial}$.

Proof. We first note that for $\xi \in C^{0,0}(\bar{M} \Theta), \xi+\bar{\xi}$ is tangent to $b M$ if and only if $*(\# \xi+\bar{\xi})=0$ on $b M$. This is easily checked in terms of admissible boundary coordinates. Thus it makes sense to speak of the diffeomorphism $e(\xi)$.

In terms of local coordinates $z=\left(z^{\prime}, \cdots, z^{n}\right)$ we can write $e(\xi)=$ $\left(f^{1}(z), \cdots, f^{n}(z)\right)$ as $f^{\alpha}(z)=z^{\alpha}+\xi^{\alpha}(z)+r^{\alpha}(z)$, where $r^{\alpha}=0\left(|\xi(z)|^{2}\right)$ is a 
$C^{\infty}$ function in $z$ and $\xi$. This follows from the definition of geodesics and standard existence and uniqueness theorems from the theory of ordinary diffierential equations. Then (2.1), for $\omega=\phi \circ e(\xi)$, becomes

$$
\begin{aligned}
& \sum_{\beta} \omega_{\alpha}^{\beta}(z)\left[\delta_{\xi}^{\gamma}+\frac{\partial \xi^{\gamma}}{\partial z^{\beta}}+\frac{\partial r^{\gamma}}{\partial z^{\beta}}+\sum_{\mu}\left(\frac{\partial \bar{\xi}^{\mu}}{\partial z^{\beta}}+\frac{\partial \bar{z}^{\mu}}{\partial z^{\beta}}\right) \varphi_{\mu}^{\gamma}(e(\xi))\right] \\
= & \frac{\partial \xi^{\gamma}}{\partial \bar{z}^{\alpha}}+\frac{\partial r^{\gamma}}{\partial \bar{z}^{\alpha}}+\sum_{\mu}\left(\delta_{\alpha}^{\mu}+\frac{\partial \bar{\xi}^{\mu}}{\partial \bar{z}^{\alpha}}+\frac{\partial \bar{r}^{\mu}}{\partial \bar{z}^{\alpha}}\right) \varphi_{\mu}^{\gamma}(e(\xi)), \quad \delta_{\beta}^{\gamma}=\left\{\begin{array}{ll}
0 & \gamma \neq \beta \\
1 & \gamma=\beta
\end{array} .\right.
\end{aligned}
$$

If $\varphi$ and $\xi$ are sufficiently small in \|\|$_{k}$-norm topology the expression in the brackets [-] represents an element of an invertible matrix $B=\left(b_{\beta}^{\gamma}\right)$. Multiplying both sides of the above equality by $B^{-1}$ we get

$$
\varphi \circ e(\xi)=\bar{\partial} \xi+\varphi+F(\varphi, \xi),
$$

where $F(\varphi, \xi)$ is of at least order 2 in $\varphi$ and $\xi$, i.e., it involves expressions which are at least quadratic in $\varphi_{\alpha}^{\beta}(e(\xi))$, $\xi^{\alpha}$ and the first order derivatives of $\xi^{\alpha}$.

Now, if $V_{1}$ and $W_{1}$ are sufficiently small neighborhoods of the origin in $\mathscr{H}_{k}^{0,1}(\Theta)$ and in the completion $\hat{P}_{k+1}$ of $P$ in the \|\|$_{k+1}$-norm topology, respectively, then it is easy to see that $j: V_{1} \times W_{1} \rightarrow \mathscr{H}_{k-1}^{0,0}(\Theta)$, $j(\varphi, \xi)=\bar{\partial}^{*}(\varphi \circ e(\xi))$, is a $C^{\infty}$ map in the sense of the theory of Banach spaces. It follows from (3.3) that the derivative of $j$ with respect to $\xi$ is the linear map $\bar{\partial} * \bar{\partial}: \widehat{P}_{k+1} \rightarrow \mathscr{H}_{k-1}^{0,0}(\Theta)$.

Since the metric $g=\sum_{\alpha, \beta} g_{\alpha \bar{\beta}} d z^{\alpha} d \bar{z}^{\beta}$ on $M^{\prime}$ is Kähler, the complex Laplacian $\square \psi$ of a scalar $(1,0)$-form $\psi=\sum_{\alpha} \psi_{\alpha} d z^{\alpha}$ is given by

$$
\square \psi=\sum_{\mu}\left(-\sum_{\alpha, \beta} g^{\alpha \bar{\beta}} \eta_{\alpha^{\prime}} \nabla_{\bar{\beta}} \psi_{\mu}\right) d z^{\mu} \text {, where } \quad\left(g^{\alpha \bar{\beta}}\right)=\left(g_{\alpha \bar{\beta}}\right)^{-1} \text {. }
$$

Also, if $\xi \in C^{0,0}(\bar{M}, \Theta)$, then

$$
\bar{\partial} * \bar{\partial} \xi=\sum_{\nu}\left(-\sum_{\alpha, \beta} g^{\alpha \bar{\beta}} \nabla_{\alpha} \nabla_{\bar{\beta}} \xi^{\nu}\right) \frac{\partial}{\partial z^{\nu}} .
$$

Now we set $\psi=\# \xi=\sum_{\mu, \nu} g_{\mu \nu} \xi^{\nu} d z^{\mu}$. Then, using the fact that the components of the metric tensor are covariant constants and the Ricci identity $\left(\nabla_{\alpha} \nabla_{\bar{\beta}}-\nabla_{\bar{\beta}} \nabla_{\alpha}\right) \xi^{\nu}=\sum_{\sigma} R_{\sigma \alpha \bar{\beta}}^{\nu} \xi^{\sigma}\left(R_{\sigma \alpha \bar{\beta}}^{\nu}=-\left(\partial \Gamma_{\sigma \alpha}^{\nu}\right) /\left(\partial \bar{z}^{\beta}\right), \Gamma_{\sigma \alpha}^{\nu}=\right.$ $\left.\sum_{r} g^{\bar{\nu}}\left(\partial g_{\bar{\gamma} \sigma}\right) /\left(\partial z^{\alpha}\right)\right)$, we obtain, by combining (3.4) and (3.5), the relation

$$
\square \sharp \xi=\# \bar{\partial} * \bar{\partial} \xi+\sum_{\mu, \sigma} R_{\mu \bar{\sigma}} \overline{\xi^{\sigma}} d z^{\mu} .
$$

Here the $R_{\mu \bar{\sigma}}$ 's are the components of the Ricci tensor $R_{\mu \bar{\sigma}}=$ $\sum_{\beta} R_{\mu \beta \bar{\sigma}}^{\beta}$. The metric $g$ is also an Einstein metric, i.e., $R_{\mu \bar{\sigma}}=R /(2 n) g_{\mu \bar{\sigma}}$ where $R=2 \sum_{\alpha} R_{\alpha}^{\alpha}=2 \sum_{\alpha, \beta} g^{\alpha \bar{\beta}} R_{\alpha \bar{\beta}}$ is the scalar curvature and $n=$ $\operatorname{dim}_{C} M=\operatorname{dim}_{c} M^{\prime}$. Thus we get that 


$$
\square \# \xi=\# \bar{\partial} * \bar{\partial} \xi+\frac{R}{2 n} \# \xi
$$

On the other hand, $\square=(1 / 2) \Delta=1 / 2\left(d d^{*}+d^{*} d\right)$. It follows from $\S 1$ that for each $C^{\infty}$ real 1 -form $\eta \in C^{1}(\bar{M})$ there exists a unique $u \in C^{1}(\bar{M})$ such that $(1 / 2) \Delta u-R /(2 n) u=\eta, * u=* d u=0$ on $b M$ and $\|u\|_{k+1} \leqq c_{k}\|\eta\|_{k-1}$. We note that by assumption $R<0$.

Now if $\psi \in C^{1,0}(\bar{M})$, then $\eta=\psi+\bar{\psi} \in C^{1}(\bar{M})$ and, conversely, every real 1-form can be represented uniquely as a sum of a scalar form of type $(1,0)$ and its conjugate. Thus we can write $u=\omega+\bar{\omega}, \omega \in C^{1,0}(\bar{M})$. Since $\Delta$ is a real operator we get, by comparing types, that for each $\xi \in C^{1,0}(\bar{M})$ there is a unique $\omega \in C^{1,0}(\bar{M})$ with

$$
\begin{gathered}
\frac{1}{2} \Delta \omega-\frac{R}{2 n} \omega=\psi, \quad *(\omega+\bar{\omega})=* d(\omega+\bar{\omega})=0, \\
\|\omega\|_{k+1} \leqq c_{k}^{\prime}\|\psi\|_{k-1}
\end{gathered}
$$

for some constant $c_{k}^{\prime}$ depending only on $k$.

Furthermore, we observe that $\sharp: C^{0,0}(\bar{M}, \Theta) \rightarrow C^{1,0}(\bar{M})$ is an isomorphism with respect to the norm \|\|$_{k}$ for any $k$. This fact and (3.8) imply that for each $\zeta \in C^{0,0}(\bar{M}, \Theta)$ there exists a unique $\xi \in P$ with $\bar{\partial} * \bar{\partial} \xi=\zeta,\|\xi\|_{k+1} \leqq \widetilde{c}_{k}\|\zeta\|_{k-1}$, for some constant $\widetilde{c}_{k}>0$.

But this shows that $\bar{\partial} * \bar{\partial}: P \rightarrow C^{0,0}(\bar{M}, \Theta)$ is an isomorphism of normed spaces. Since $P$ and $C^{0,0}(\bar{M}, \Theta)$ are dense in $\hat{P}_{k+1}$ and $\mathscr{H}_{k-1}^{0,0}(\Theta)$, respectively, $\bar{\partial} * \bar{\partial}: \hat{P}_{k+1} \rightarrow \mathscr{K}_{k-1}^{0,0}(\Theta)$ is an isomorphism of Banach spaces. By the implicit function theorem it follows that there exists a neighborhood $V$ of the origin in $\mathscr{H}_{k}^{0,1}(\Theta)$ and a $C^{\infty}$ map $\xi: V \rightarrow W_{1}$ such that $\bar{\partial}^{*}\left(\varphi \circ e(\xi(\varphi))=0\right.$. This equality can also be written as $\bar{\partial} * \bar{\partial} \xi(\varphi)+\bar{\partial}^{*} \varphi+$ $\bar{\partial}^{*} F(\varphi, \xi(\varphi))=0$, which is a second-order elliptic differential equation with respect to $\xi$. Thus $\xi(\varphi) \in C^{0,0}(\bar{M}, \Theta)$ whenever $\varphi \in C^{0,1}(\bar{M}, \Theta)$.

We now consider the covariant differentiation operator $D=\tilde{\partial}+\bar{\partial}$ on $C^{p, q}(\bar{M}, \Theta)$. Here $\tilde{\partial} \varphi=\partial \varphi+\theta \wedge \varphi$ and $\theta$ is the connection of the metric $g$. Since $g$ is Kähler we have that

$$
\begin{gathered}
\tilde{\Delta}=D D^{*}+D^{*} D=\square^{\prime}+\square^{\prime \prime}, \text { where } \square^{\prime}=\bar{\partial} \bar{\partial}^{*}+\bar{\partial}^{*} \bar{\partial}, \quad \square^{\prime \prime} \\
=\tilde{\partial} \tilde{\partial}^{*}+\tilde{\partial} * \tilde{\partial}, \quad \tilde{\partial}^{*}=-* \tilde{\partial} * \\
\tilde{A}=\square^{\prime}-\square^{\prime \prime}=\sqrt{-1}(e(s) \Lambda-\Lambda e(s)),
\end{gathered}
$$

where $e(s) \varphi=\bar{\partial} \theta \wedge \varphi$ and $\Lambda \varphi=*^{-1}(\rho \wedge * \varphi)$ with $\rho=\sqrt{-1} \sum_{\alpha, \beta} g_{\alpha \bar{\beta}} d z^{\alpha} \wedge$ $d \bar{z}^{\beta}$.

We refer to [2] for verification of the above identities.

In addition to the Kähler-Einstein condition on the metric $g$ we also make the following assumption: 
There exists a constant $c>0$ such that for all $\varphi \in C^{0, q}(\bar{M}, \Theta), \quad q=1,2, \quad(\widetilde{A} \varphi, \varphi) \geqq c\|\varphi\|^{2}$.

A large class of Kähler-Einstein manifolds of negative scalar curvature satisfying (3.11) is considered in [1].

It follows from (3.9) and (3.10) that $\square^{\prime}=1 / 2(\tilde{J}+\widetilde{A})$. Then (3.11) together with the results of $\S 1$ imply that for each $\varphi \in C^{0, q}(\bar{M}, \Theta)$, $q=1,2$, there exists a unique $\psi \in C^{0, q}(\bar{M}, \Theta)$ with $* \psi=* D \psi=0$ on $b M, \square^{\prime} \psi=\varphi$ and $\|\psi\|_{k+2} \leqq$ const. $\|\varphi\|_{k}$. In the sequel we will denote $\psi$ by $G \varphi$. Moreover, $\varphi$ can be uniquely represented as $\varphi=\varphi_{1}+\varphi_{2}$ where $\varphi_{1}=G \square^{\prime} \varphi$ and $\square^{\prime} \varphi_{2}=0$. We set $H \varphi=\varphi_{2}$. Similarly, using the notation introduced in Proposition 3.6, for each $\xi \in C^{0,0}(\bar{M}, \Theta)$ there exists a unique $\zeta \in P$ (denoted by $G \xi$ ) such that $\bar{\partial} * \bar{\partial} G \xi=\xi$, $\|G \xi\|_{k+2} \leqq$ const. $\|\xi\|_{k}$. Also, $\xi$ can be uniquely represented as $\xi=\xi_{1}+\xi_{2}$ where $\xi_{1}=G \bar{\partial} * \bar{\partial} \xi$ and $\bar{\partial} * \bar{\partial} \xi_{2}=0$. Again we set $H \xi=\xi_{2}$. The use of the same letters $G$ and $H$ for the spaces $C^{0, q}(\bar{M}, \Theta)$ and $C^{0,0}(\bar{M}, \Theta)$ will not cause any confusion.

We are now ready to prove our main result.

Theorem 3.7 (Main Theorem). Let $M^{\prime}$ be a complex KählerEinstein manifold of negative scalar curvature. Let $M$ be a subset of $M^{\prime}$ with nonempty interior such that its boundary $b M$ is a $C^{\infty}$ submanifold of $M^{\prime}$ of (real) dimension $(2 n-1)\left(n=\operatorname{dim}_{C} M\right), \bar{M}=$ $M \cup b M$ is compact in $M^{\prime}$, and condition (3.11) is satisfied. Then a universal family of deformations of $M$ exists.

Proof. Proposition 3.6 suggests that it is natural to construct a universal family by considering the set $\Phi=\left\{\phi \in C^{0,1}(\bar{M}, \Theta) \mid \bar{\partial} \varphi-\right.$ $\left.[\varphi, \varphi]=0, \bar{\partial}^{*} \varphi=0\right\}$.

If $\varphi \in \Phi$, then $\varphi-G \bar{\partial} *[\varphi, \varphi]=H \varphi$ and thus

$$
\Phi \subset \Psi=\left\{\varphi \in C^{0,1}(\bar{M}, \Theta) \mid \varphi-G \bar{\partial}^{*}[\varphi, \varphi] \in H^{0,1}\right\}
$$

where $H^{0,1}=\left\{\varphi \in C^{0,1}(\bar{M}, \Theta) \mid \square^{\prime} \varphi=0\right\}$.

The $\operatorname{map} J: C^{0,1}(\bar{M}, \Theta) \rightarrow C^{0,1}(\bar{M}, \Theta)$ given by $J \varphi=\varphi-G \overline{\partial^{*}}[\varphi, \varphi]$ is uniformly continuous in \|\|$_{k}$-norm topology and therefore it can be considered as a continuous map $J: \mathscr{H}_{k}^{0,1}(\Theta) \rightarrow \mathscr{K}_{k}^{0,1}(\Theta)$. Since the operators $G$ and $\bar{\partial}^{*}$ are linear and the bracket is bilinear, $J$ is also complex analytic. The differential of $J$ at the origin is the identity map. Hence, by the inverse mapping theorem for Banach spaces there exists a neighborhood $N$ of 0 in $\mathscr{H}_{k}^{0,1}(\Theta)$ and a complex analytic map $t \mapsto \varphi(t), t \in N$, such that $J(\varphi(t))=t$. Let $N^{\prime}=N \cap H^{0,1}$. Then, for $t \in N^{\prime}, \varphi(t)-G \bar{\partial}^{*}[\varphi(t), \varphi(t)]=t$ and by applying the operator $\square^{\prime}$ we get that $\square^{\prime} \varphi(t)-\bar{\partial}^{*}[\varphi(t), \varphi(t)]=0$. Hence, $\varphi(t) \in C^{0,1}(\bar{M}, \Theta)$ if $t \in N^{\prime}$. Moreover, $\varphi(0)=0$ and if $t$ is sufficiently small in \|\|$_{k}$-norm, $\varphi(t)$ 
represents an almost complex structure $M_{\varphi(t)}$ on $\boldsymbol{M}$.

We now give a necessary and sufficient condition on $t$ so that $\varphi(t) \in \Phi$.

Since $J(\varphi(t))=t, \bar{\partial} \varphi(t)-\bar{\partial} G \bar{\partial}^{*}[\varphi(t),(t)]=\bar{\partial} t$. By using the relations $\bar{\partial} G \psi=G \bar{\partial} \psi+H \bar{\partial} G \dot{\psi}$ and $\square^{\prime} G \omega=G \square^{\prime} \omega-H \omega$, $\psi \in C^{0,1}(\bar{M}, \Theta)$, $\omega \in C^{0, q}(\bar{M}, \Theta), q=0,1$, we obtain the equality

$$
\begin{aligned}
& \bar{\partial} \varphi(t)-[\varphi(t), \varphi(t)] \\
= & \bar{\partial} t-G \bar{\partial} * \bar{\partial}[\varphi(t), \varphi(t)]-H[\varphi(t), \varphi(t)]+H \bar{\partial} G \bar{\partial}^{*}[\varphi(t), \varphi(t)] .
\end{aligned}
$$

Let $\omega(t)$ be the right-hand side of (3.12). Since the operators $G, H, \bar{\partial}, \bar{\partial}^{*}$ are linear operators and [,] is bilinear $\omega: N^{\prime} \rightarrow \mathscr{L}_{k=1}^{0,2}(\Theta)$ is complex analytic. Hence, $\varphi(t) \in \Phi$ if and only if $t \in T=T_{1} \cap T_{2}$, where $T_{1}=\left\{t \in N^{\prime} \mid \omega(t)=0\right\}$ and $T_{2}=\left\{t \in N^{\prime} \mid \bar{\partial}^{*} \varphi(t)=0\right\}$. Moreover, $T$ is a Banach analytic set and the map $\varphi: T \rightarrow C^{0,1}(\bar{M}, \Theta), t \mapsto \varphi(t)$, is analytic. Thus $(T, \varphi, 0)$ represents a complex analytic family of deformations of $M$. We will now prove that this family is univarsal.

Let $\left(S, \psi, s_{0}\right)$ be any other family of deformations of $M$. If $S^{\prime}$ is a sufficiently small neighborhood of $s_{0}$, then, by Proposition 3.6, for each $\psi(s)$ there exists a unique $\xi(s) \in P$ such that $\bar{\partial}^{*}(\psi(s) \circ e(\xi(e)))=0$. Thus $\psi(s) \circ e(\xi(s)) \in \Phi$, so there is a map $\tau: S^{\prime} \rightarrow T$ such that $\psi(s) \circ e(\xi(s))=$ $\varphi(\tau(s))$. It only remains to show that $\tau$ is complex analytic.

We first observe that the proof of Proposition 3.6 also gives the $C^{\infty}$ dependence of $\xi$ on $s$. Next, $\varphi(\tau(s))-G \bar{\partial} *[\varphi(\tau(s))$, $\varphi(\tau(s))]=\tau(s)$ and $\tau(s) \in H^{0,1}$. Therefore $\tau(s)=H p(\tau(s))=H(\psi(s) \circ e(\xi(s)))$. By definition, at each $s \in S^{\prime}, \psi(s)$ can be extended to an analytic map of a neighborhood $U$ of $s$ in the ambiant space of $S$. Then $\xi(s)$ has a $C^{\infty}$ extension in such $U^{\prime}$ s so that $\bar{\partial}^{*}(\psi(s) \circ e(\xi(s)))=0$. Since $\tau(s)=$ $H(\psi(s) \circ e(\xi(s)))$ for points on $S^{\prime}$, it is clear that $\tau(s)$ can also be extended to a $C^{\infty}$ mapping on a neighborhood $U$ of $s$ in the ambiant space of $S$. We will actually show that $\tau(s)$ is complex analytic on $U$ if $S^{\prime}$ is a sufficiently small neighborhood of $s_{0}$ in $S$. Therefore, without loss of generality, we may assume that $U$ is an open subset of a finite dimensional complex space. For each $s, u \in U$ we have $\psi(s+u) \circ e(\xi(s+u))=\psi(s+u) \circ e(\xi(s))-\psi(s) \circ e(\xi(s))+K+\psi(s) \circ e(\xi(s+u))$, where

$$
\begin{aligned}
K= & \psi(s+u) \circ e(\xi(s+u))-\psi(s+u) \circ e(\xi(s)) \\
& +\psi(s) \circ e(\xi(s))-\psi(s) \circ e(\xi(s+u)) .
\end{aligned}
$$

Since $\psi \circ e(\xi)=\psi+\bar{\partial} \xi+F(\dot{\xi}, \xi)$, we find that $K=0\left(|u|^{2}\right)$. The map $\tau$ will be complex analytic on $U$ if we can show that for each tangent vector $\ell$ of type $(0,1), l[\psi(s+u) \circ e(\xi(s+u))]_{u=0}=0$. First we observe that for fixed $s, \psi(s+u) \circ e(\xi(s))$ depends complex analytically on $u$. Next by Proposition 2.5, we have that 


$$
\begin{gathered}
\psi(s) \circ e(\xi(s+u))=\psi(s)+\bar{\partial}_{\psi(s)}(\xi(s+u)+\psi(s) \circ \overline{\xi(s+u))} \\
+\left(\bar{\partial} \psi(s)-[\psi(s), \psi(s)] \circ \xi(s+u)+0\left(|u|^{2}\right) .\right.
\end{gathered}
$$

Therefore,

$$
\begin{aligned}
& \ell[\psi(s+u) \circ e(\xi(s+u))]_{u=0} \\
= & \bar{\partial}_{\psi(s)}(\ell \xi+\psi(s) \circ \overline{\ell \xi})+(\bar{\partial} \psi(s)-[\psi(s), \psi(s)]) \circ \overline{\ell \xi} \\
& \text { where } \ell \xi=\ell[\xi(s+u)]_{u=0} .
\end{aligned}
$$

We apply the operator $G \bar{\partial}^{*}$ to both sides of (3.15). We note that since $\ell \xi \in P G \bar{\partial} * \bar{\partial} \ell \xi=\ell \xi$. This, together with the fact that $\bar{\partial}^{*}(\psi(s+$ $u) \circ e(\xi(s+u)))=0$, implies that

$$
\begin{gathered}
\ell \xi=G \bar{\partial} *[\psi(s), \iota \xi]-G \bar{\partial}^{*} \bar{\partial}_{\psi(s)}(\psi(s) \circ \overline{\ell \xi}) \\
-G \bar{\partial} *(\bar{\partial} \psi(s)-[\psi(s), \psi(s)]) \circ \overline{\ell \xi} .
\end{gathered}
$$

We estimate (3.16) in \|\|$_{k}$-norm and obtain the inequality

$$
\|\ell \xi\|_{k} \leqq \text { const. }\|\psi(s)\|_{k} \cdot\|\ell \xi\|_{k} \text {. }
$$

Since $\psi\left(s_{0}\right)=0$, const. $\|\psi(s)\|_{k}<1$ if $S^{\prime}$ is sufficiently small. But then (3.17) is satisfied if and only if $\ell \xi=0$. Hence, $\ell[\tau(s+u)]_{u=0}=$ $H \ell[\psi(s+u) \circ e(\xi(s+u))]_{u=0}=0$. Therefore, the map $\tau$ is complex analytic.

4. Compact Riemann surfaces with boundary. It is natural to ask the following question: By introducing suitable boundary conditions on the elements of $C^{0,1}(\bar{M}, \Theta)$ can one find a category of families of complex structures of $M$ such that the universal family is parametrized by a finite dimensional space? We will answer this question affirmatively in the case of bordered compact Riemann surfaces $M$. At the same time, without making use of quasiconformal mappings and Schwarzian derivatives, we will describe sufficiently small complex structures on $M$ in terms of the reduced Teichmüller space considered in detail in [3] and [4].

Let $M^{\prime}$ be the double of $M$, i.e., if, for some Fuchsian group $\Gamma, M=U / \Gamma$, then $M^{\prime}=\Omega / \Gamma$, where $U$ is the upper half plane of the complex plane $C$ and $\Omega$ is the complement of the limit set of $\Gamma$. The symmetry $J$ of $M^{\prime}$, changing the orientation of $M^{\prime}$, is an anticonformal involution and can be lifted to the involution $z \mapsto \bar{z}$ of $\boldsymbol{C}$. It is shown in [3] that there is a bijective correspondence between $C^{0,1}(\bar{M}, \Theta)$ and $C_{r}^{0,1}\left(M^{\prime}, \Theta\right)=\left\{\varphi \in C^{0,1}\left(M^{\prime}, \Theta\right) \mid \varphi\right.$ commutes with $\left.J\right\}$, i.e., if $\varphi=\mu(z) d \bar{z}(\partial / \partial z)$ then, on $\Omega, \overline{\mu(z)}=\mu(\bar{z})$. Let $T=\left\{\varphi \in C_{r}^{0,1}\left(M^{\prime}, \Theta\right) \mid \varphi=\right.$ $\left.\lambda^{-2} \bar{\nu} d \bar{z}(\partial / \partial z), \sup _{M^{\prime}}\left|\lambda^{-2} \bar{\nu}\right|<1\right\}$ where $\lambda^{2} d z d \bar{z}$ is the Poincaré metric on $M^{\prime}$ and $\nu d z^{2}$ is a holomorphic quadratic differential. It is well known (see [4]) that $T$ is a finite dimensional real vector space. Let $\mathscr{K}_{r, k}^{0,1}(\Theta)$ be the completion of $C_{r}^{0,1}\left(M^{\prime}, \Theta\right)$ is the \|\|$_{k}$-norm. 
THEOREM 4.1. The space $T$ represents a universal family of deformations of $M$ in the category of real analytic families of deformations of $M$, i.e., if $\left(S, \psi, s_{0}\right)$ is given with $S$ an open set of a (real) finite dimensional Euclidean space, $\psi: S \rightarrow C_{r}^{0,1}\left(M^{\prime}, \Theta\right)$ a real analytic map ( $\psi$ composed with the inclusion $C_{r}^{0,1}\left(M^{\prime}, \Theta\right) \subset \mathscr{C}_{r, k}^{0,1}(\Theta)$ is an analytic map of real Banach spaces), $\sup _{\bar{M}}|\mu(z, s)|<1, \psi(z, s)=$ $\mu(z, s) d \bar{z}(\partial / \partial z)$, and $\psi\left(s_{0}\right)=0$ then there exists a real analytic map $\tau: S \rightarrow T$ with $\tau\left(s_{0}\right)=0$.

Proof. We first note that each $\varphi=\mu(z) d \bar{z}(\partial / \partial z) \in C^{0,1}(\bar{M}, \Theta)$ with $\sup _{\bar{M}}|\mu(z)|<1$ represents an almost comlex structure on $M$ and thus a complex structure since $\bar{\partial} \varphi=[\varphi, \varphi]=0$ on a Riemann surface. Next, we observe that $M^{\prime}$ is a Kähler-Einstein manifold of negative (scalar) curvature. Thus, by Proposition 3.6, if $\|\varphi\|_{k}$ is sufficiently small then there exists a unique $\xi=\xi(\varphi) \in P$ such that $\bar{\partial}^{*}(\varphi \circ e(\xi))=0$. Now, if $f=e(\xi)$ and $d_{p} f$ is the differential of $f$ at $p \in b M$, then

$$
\begin{gathered}
d_{p} f(\eta) \| b M \text { for any real vector } \eta \| b M \\
d_{p} f(\zeta) \perp b M \text { for any real vector } \zeta \perp b M .
\end{gathered}
$$

The first statement follows from the fact that $f$ carries $b M$ onto $b M$. For the second statement we make, as in [7], the following observation. Let $b(t), 0 \leqq t \leqq t_{0}=\|\xi+\bar{\xi}\|_{p}$, be the geodesic with $b(0)=p, b\left(t_{0}\right)=f(p)$ and $\dot{b}(0)=(\xi(p)+\bar{\xi}(p)) /\left(\|\xi+\bar{\xi}\|_{p}\right)$. We note that the construction of $f$ given in $\S 3$ implies $b(t) \subset b M$. Then $d_{p} f(\zeta)=$ $v\left(t_{0}\right)$, where $v(t)$ is the Jacobi field along $b(t)$ with $v(0)=\zeta$ and $v^{\prime}(0)=\nabla_{\zeta}(\xi+\bar{\xi})=\left(\zeta^{1} \nabla_{x} u^{1}+\zeta^{2} \nabla_{y} u^{1}\right) \partial /(\partial x)+\left(\zeta^{1} \nabla_{x} u^{2}+\zeta^{2} \nabla_{y} u^{2}\right) \partial /(\partial y)$ where $\zeta=\zeta^{1}(\partial / \partial x)+\zeta^{2}(\partial / \partial y), \quad \xi=\left(u^{1}+i u^{2}\right) \partial /(\partial z)$ and $\nabla_{x}$ and $\nabla_{y}$ represent covariant derivatives. It is easy to check, in terms of boundary coordinates, that if $\zeta \perp b M$ at $p=b(0)$ then $v^{\prime}(0) \perp b M$ at $p$ since the condition $\zeta \in P$ implies that $\nabla_{x} u^{2}=\nabla_{y} u^{1}=0$ on $b(t)$. Then it follows from the properties of Jacobi fields that $v(t) \perp b(t)$ for each $t, 0 \leqq$ $t \leqq t_{0}$. Hence, at $t=t_{0}, d_{p} f(\zeta) \perp b M$.

Next, (4.1) and (4.2) imply that, in terms of boundary coordinates, $f_{z}$ and $f_{\bar{z}}$ are real on $b M$. Now, if $\varphi \in C_{r}^{0,1}\left(M^{\prime}, \Theta\right)$ then $\varphi$ is real on $b M$ and, therefore, $\varphi \circ e(\xi)=\left(f_{\bar{z}}+\varphi(f(z)) \bar{f}_{\bar{z}}\right) /\left(f_{z}+\varphi(f(z)) \bar{f}_{z}\right)$ is real on $b M$. On the other hand, $\bar{\partial}^{*} \omega=0$ for $\omega \in C^{0} \cdot{ }^{1}(\bar{M}, \Theta)$ if and only if $\omega=\lambda^{-2} \bar{\nu} d \bar{z}(\partial / \partial z)$ where $\nu d z^{2}$ is a holomorphic quadratic differential on $M$. In our case, $\omega=\rho \circ e(\xi)$ and hence $\nu$ is real on $b M$. Since $M^{\prime}$ is symmetric with respect to $b M, \nu d z^{2}$ can be extended to a holomorphic quadratic differential on $M^{\prime}$. Therefore, $\varphi \in C_{r}^{0,1}\left(M^{\prime}, \Theta\right)$ implies that $\varphi \circ e(\xi) \in T$.

If $\left(S, \psi, s_{0}\right)$ is any real analytic family of deformations of $M$, the arguments given above show that there is a $C^{\infty}$ map $\tau: S^{\prime} \rightarrow T$ where 
$S^{\prime}$ is a neighborhood of $s_{0}$. Let $S^{\prime}$ be an open subset of $R^{m}$ and let $S^{*}$ be an open subset of $C^{m}$ such that $S^{*} \cap \boldsymbol{R}^{m}=S^{\prime}$ and $\psi(s)$ has a complex analytic extension $\psi^{*}: S^{*} \rightarrow C^{0,1}\left(M^{\prime}, \Theta\right)$. By Proposition 3.6 the $C^{\infty}$ family of vector fields $\xi(s) \in P, s \in S^{\prime}$, with $\tau(s)=\psi(s) \circ e(\xi(s))$ can be extended to a $C^{\infty}$ family $\xi\left(s^{*}\right) \in P, \mathrm{~s}^{*} \in S^{*}$ such that $\bar{\partial}^{*}\left(\psi\left(s^{*}\right) \circ e\left(\xi\left(s^{*}\right)\right)=0\right.$. Therefore, $\tau$ can be extended to a $C^{\infty}$ map $\tau^{*}: S^{*} \rightarrow T^{*}=\left\{\omega \in C^{0,1}(\bar{M}, \Theta) \mid \bar{\partial}^{*} \omega=0\right\}$. It follows from the proof of Theorem 3.7 that $\tau^{*}$ is complex analytic if $S^{\prime}$ is a sufficiently small neighborhood of $s_{0}$. Hence $\tau: S^{\prime} \rightarrow T$ is real analytic.

\section{REFERENCES}

1. A. Andreotti and E. Vesentini, On deformations of discontinuous groups, Acta Math., 112 (1964), 249-298.

2. E. Calabi and E. Vesentini, On compact, locally symmetric Kähler manifolds, Ann. of Math., 71 (1960), 472-507.

3. C. J. Earle, Teichmüller spaces of groups of the second kind, Acta Math., 112 (1964), 91-97.

4. - Reduced Teichmüller spaces, Trans. Amer. Math. Soc., 126 (1967), 54-63.

5. G. Fichera, Linear Elliptic Differential Systems and Eigenvalue Problems, Springer-Verlag, Berlin-Heidelberg-New York, 1965.

6. G. Kiremidjian, Complex structures on Riemann surfaces, Trans. Amer. Math. Soc., 169 (1972), 317-336.

7. - Deformations of complex structures on certain noncompact manifolds, Ann. of Math., 98, No. 3 (1973), 411-426.

8. M. Kuranishi, New Proof for the Existence of Locally Complete Families of Complex Structures, Proc. Conf. Complex Analysis (Minneapolis, 1964), Springer, Berlin, 1965, 142-154.

9. - Deformations of complex analytic structures on compact manifolds, Seminar on Global Analysis, Montreal, 1969.

10. C. B. Morrey, Jr., Multiple Integrals in the Calculus of Variations, Springer, 1966.

11. L. Nirenberg, Remarks on strongly elliptic partial differential equations, Comm. Pure Appl. Math., 8 (1955), 648-674.

Received April 5, 1973. This research is supported in part by grants from the National Science Foundation, GP-33942X and P019572-002.

StANFORd UNIVERSITY 


\section{PACIFIC JOURNAL OF MATHEMATICS}

\section{EDITORS}

RICHARD ARENS (Managing Editor)

University of California

Los Angeles, California 90024

R. A. Beaumont

University of Washington

Seattle, Washington 98105
J. DugundJI

Department of Mathematics University of Southern California Los Angeles, California 90007

D. Gilbarg AND J. Milgram Stanford University

Stanford, California 94305

\section{ASSOCIATE EDITORS}
E. F. BECKENBACH
B. H. NeUmanN
F. WOLF
K. YOSHIDA

\section{SUPPORTING INSTITUTIONS}

UNIVERSITY OF BRITISH COLUMBIA CALIFORNIA INSTITUTE OF TECHNOLOGY

UNIVERSITY OF CALIFORNIA

MONTANA STATE UNIVERSITY

UNIVERSITY OF NEVADA

NEW MEXICO STATE UNIVERSITY

OREGON STATE UNIVERSITY

UNIVERSITY OF OREGON

OSAKA UNIVERSITY
UNIVERSITY OF SOUTHERN CALIFOF

STANFORD UNIVERSITY

UNIVERSITY OF TOKYO

UNIVERSITY OF UTAH

WASHINGTON STATE UNIVERSITY UNIVERSITY OF WASHINGTON

AMERICAN MATHEMATICAL SOCIET NAVAL WEAPONS CENTER 


\section{Pacific Journal of Mathematics}

\section{Vol. 54, No. 1 \\ May, 1974}

Ralph K Amayo, Engel Lie rings with chain conditions ..................

Bernd Anger and Jörn Lembcke, Hahn-Banach type theorems for hypolinear

functionals on preordered topological vector spaces ..................

Gregory Frank Bachelis and Samuel Ebenstein, On $\Lambda(p)$ sets ................

Harvey Isaac Blau, Indecomposable modules for direct products of finite

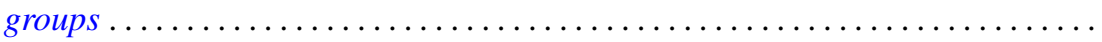

Larry Eugene Bobisud and James Calvert, Singular perturbation of a

time-dependent Cauchy problem in a Hilbert space ................

Walter D. Burgess and Robert Raphael, Abian's order relation and orthogonal

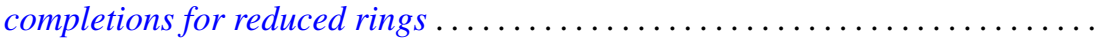

James Diederich, Representation of superharmonic functions mean continuous at

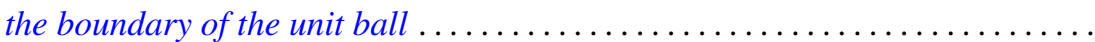

Aad Dijksma and Hendrik S. V. de Snoo, Self-adjoint extensions of symmetric

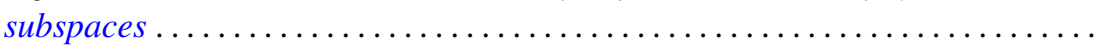

Gustave Adam Efroymson, A Nullstellensatz for Nash rings . . . . . . . . . . . . .

John D. Elwin and Donald R. Short, Branched immersions onto compact orientable surfaces . . . . . . . . . . . . . . . . . . . . . . . . .

John Douglas Faires, Comparison of the states of closed linear

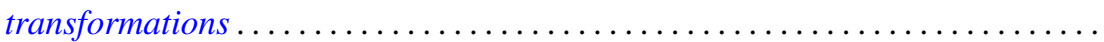

Joe Wayne Fisher and Robert L. Snider, On the von Neumann regularity of rings with regular prime factor rings .

Franklin Takashi Iha, A unified approach to boundary value problems on compact intervals

Palaniappan L. Kannappan and Che Tat $\mathrm{Ng}$, On functional equations connected with directed divergence, inaccuracy and generalized directed divergence

Samir A. Khabbaz and Elias Hanna Toubassi, The module structure of Ext $(F, T)$ over the endomorphism ring of $T$...

Garo K. Kiremidjian, On deformations of complex compact manifolds with boundary.

Dimitri Koutroufiotis, Mappings by parallel normals preserving principal

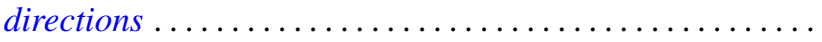

W. K. Nicholson, Semiperfect rings with abelian adjoint group

Norman R. Reilly, Extension of congruences and homomorphisms to translational hulls

Sadahiro Saeki, Symmetric maximal ideals in $M(G)$

Brian Kirkwood Schmidt, On the homotopy invariance of certain functors ...

H. J. Shyr and T. M. Viswanathan, On the radicals of lattice-ordered rings ...

Indranand Sinha, Certain representations of infinite group algebras ...

David Smallen, The group of self-equivalences of certain complexes ...

Kalathoor Varadarajan, On a certain problem of realization in homotopy

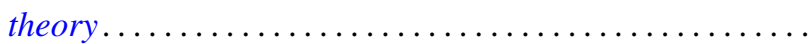

